\title{
Rapidly increasing incidence in scoliosis surgery over 14 years in a nationwide sample
}

\author{
Johan von Heideken $^{1}$ - Maura D. Iversen ${ }^{1,2,3} \cdot$ Paul Gerdhem $^{4,5}$
}

Received: 17 June 2017 / Revised: 13 August 2017 / Accepted: 10 October 2017 / Published online: 19 October 2017

(C) The Author(s) 2017. This article is an open access publication

\begin{abstract}
Purpose Severe scoliosis is primarily managed with surgery. This cohort study describes the incidence of surgically treated scoliosis among Swedish youth and young adults, stratified by age, sex, scoliosis type, and surgical approach and identifies changes in incidence rate and hospital length of stay (LOS), infections requiring re-surgery and mortality within 90 days.

Methods Swedish youth, 0-21 years, $(n=3062)$ with a diagnostic code for scoliosis and spine surgery between 2000 and 2013 were selected from the National Patient Register. Incidence was computed by comparing individuals with surgically treated scoliosis to the total at risk population. Linear regression models and Spearman correlation coefficients analyzed trends over time.
\end{abstract}

Paul Gerdhem

paul.gerdhem@sll.se

1 Department of Women's and Children's Health, Karolinska Institutet, Karolinska Universitetssjukhuset Solna, 17176 Stockholm, Sweden

2 Department of Physical Therapy, Movement and Rehabilitation Sciences, Northeastern University, 360 Huntington Avenue, Rm 301c Robinson Hall, Boston, MA 02115, USA

3 Section of Clinical Sciences, Department of Medicine, Harvard Medical School, Brigham and Women's Hospital, Boston, MA, USA

4 Department of Clinical Science, Intervention and Technology, Division of Orthopedics and Biotechnology, Karolinska Institutet, Karolinska University Hospital, Huddinge, K54, 14186 Stockholm, Sweden

5 Department of Orthopaedics, Karolinska University Hospital, Stockholm, Sweden
Results Overall annual incidence per 100,000 individuals was 9.1 (5.9 males/12.5 females). Annual incidence increased over 14 years from 5.1 to 9.8 ; an average $4.6 \%$ per year $(p<0.001)$. Adolescent idiopathic scoliosis was most common ( 4.5 per 100,$000 ; n=1516$ ) followed by neuromuscular $2.7(n=913)$ and congenital $0.7(n=236)$. Average LOS decreased among scoliosis types except infantile and neuromuscular scoliosis. Posterior fusion was the most common surgical approach (75\%) followed by anterior (18\%) and anteroposterior fusion (7\%). Posterior fusions significantly increased with a resultant decrease in anterior and anteroposterior fusion over time. Individuals with neuromuscular scoliosis exhibited the highest mortality $(n=12 ; 1.3 \%)$ and ( $n=59 ; 6 \%)$ of individuals with neuromuscular scoliosis and $(n=12 ; 15 \%)$ with scoliosis related to MMC required revision surgery due to post-op infection.

Conclusions Surgical management of scoliosis is increasing with a concurrent decrease in hospital LOS. Surgical management of neuromuscular scoliosis is associated with high 90-day post-operative infections and mortality rate.

Keywords Spine $\cdot$ Children · Young adults · Surgery · Epidemiology

\section{Introduction}

Scoliosis affects roughly $3 \%$ of children and adolescents in Southern Sweden [1]. Of these individuals, in approximately $85 \%$ there is no known etiology, and therefore, the scoliosis diagnosis is referred to as idiopathic scoliosis [1,2]. There are a number of non-idiopathic forms of scoliosis including: congenital scoliosis, resulting from embryological defects in vertebral development, and neuromuscular scoliosis, which may develop in individuals diagnosed with neurological or 
muscular disorders. Neurological or muscular disorders that may lead to scoliosis are cerebral palsy, myelomeningocele (MMC), muscular dystrophies, and spinal muscular atrophies [3-5].

The main focus of medical intervention for scoliosis is primarily to avert curve progression and secondarily, to correct the curve should the curve progress. In $10 \%$ of scoliosis cases the curvature becomes severe [1] and is associated with respiratory issues, disability and pain. Severe curvature in the presence of, or risk of, compromised health is an indication for surgical intervention. Clinical decision-making regarding surgery for scoliosis includes: the individual's age, curve progression, symptoms and any significant underlying disorders $[1,3,5,6]$.

Presently, we are not aware of any studies that have described the incidence of surgical treatment for different types of pediatric scoliosis and few studies have examined incidence of surgery for various forms of scoliosis by sex. There is also limited information on trends in length of stay $[7,8]$. This study will examine data on patients surgically treated for scoliosis in Sweden between 2000 and 2013 and aims to answer the following questions:

1) What is the true incidence and change in incidence of surgically treated scoliosis among Swedish individuals (ages $0-21$ years), what are the diagnoses, and in which age groups is surgery performed?

2) How did the incidence for various scoliosis diagnoses differ by sex?

3) What are the trends in hospital length of stay (LOS), infection rates requiring revision surgery and mortality rates within 90 days, and how do these differ according to diagnoses?

\section{Materials and methods}

The Ethical Review Board in Stockholm approved the study (number 2012/172-31/4). We hypothesized that trends in surgical rates for children with scoliosis would vary over time and by type of scoliosis and gender. We further hypothesized that infection rates, mortality rates, and LOS would decrease over time. We gathered data from the National Patient Register [9] which codes diagnoses according to the International Classification of Diseases ICD-10 [10] and includes every individual hospitalized in Sweden. Each Swedish individual can be identified by a unique 10-digit national registration number, making it possible to retrieve demographic data, as well as data on type of scoliosis, surgical approach, surgical revisions due to post-operative infections, and duration of hospital stay. We identified all youth and young adults aged 0-21 admitted for primary scoliosis surgery, between January 1 ,
1997, through December 31, 2013, using diagnostic codes: M41*, Q05*, Q76* (* indicates all of the 4th and 5th characters) and surgical codes for spine surgery: NAT19, NAT29, NAG39, NAG49, NAG59, NAG 69, NAG79, NAG89 and for surgery due to infections within 90 days: NAS19, NAS29, NAS49, NAS59, NAS69, NAS99, NAU89, NAW59, NAW69, QWB, T81.4, and T84.6 [11]. To minimize the possibility of including children who had scoliosis surgery prior to the study period, we used a "wash out" period from 1997 to 2000, and only included children from January 1, 2000 to December 31, 2013 in our study population.

Type of scoliosis was stratified into: infantile idiopathic scoliosis, juvenile idiopathic scoliosis, adolescent idiopathic scoliosis, neuromuscular scoliosis, scoliosis related to MMC, congenital scoliosis, and scoliosis unspecified (including other secondary scoliosis and other forms of scoliosis). We examined the proportion children who developed infections leading to a revision surgery within 90 days (for each type of scoliosis) and the proportion of children who had infections during the first 7 years of the study was compared to the last 7 years. Of these 3062 individuals, 1017 (33\%) were males and 2045 (67\%) females between the ages of 0-21 years, admitted for inpatient surgery for scoliosis from 2000 to 2013 (Table 1). These data were linked with the Cause of Death Registry to retrieve date of death to identify mortality within 90 days from the primary scoliosis surgery [12].

\section{Statistical analysis}

Annual incidence was computed by comparing the number of individuals with surgically treated scoliosis to the total number of individuals at risk. Total number of individuals at risk was calculated by summing up the year population (0-21 years of age) for each year (2000-2013), in total $33,585,397$ (17,240,398 males and 16,344,999 females) person years), stratified by year of primary surgery, and sex. The number of individuals at risk was provided by government census data [13]. Descriptive statistics, such as counts, percentage, median with interquartile range (IQR) and cross tabulation were used to analyze data on surgical approach, length of hospital stay (LOS), post-operative infections needing surgical revision and mortality within 90 days. We used relative risks (RR) to examine the impact of revision surgery pre and post 2006 (due to changes in materials used for implant; stainless steel versus titanium alloy). Trends over time for primary outcomes were analyzed with linear regression models and Spearman rank correlation coefficients. Statistical significance was defined as $p<0.05$. All statistical analyses were performed using SPSS for Mac 23.0 (SPSS Inc., Chicago, IL). 
Table 1 Number of admissions and median age for primary scoliosis surgery for individuals aged 0-21 years in Sweden (2000 through 2013), stratified by sex

\begin{tabular}{|c|c|c|c|c|c|c|}
\hline Type of Scoliosis & Males $N(\%)$ & Age years (IQR) & Females $N(\%)$ & Age years (IQR) & Total $N(\%)$ & Age years (IQR) \\
\hline Regardless of type & $1017(33)$ & $15(13-17)$ & $2045(67)$ & $14(12-16)$ & $3052(100)$ & $15(13-16)$ \\
\hline Infantile idiopathic & $4(36)$ & $10(4-17)$ & $7(64)$ & $6(2-9)$ & $11(0)$ & $6(2-10)$ \\
\hline Juvenile idiopathic & $54(24)$ & $15(13-17)$ & $176(76)$ & $14(13-16)$ & $230(8)$ & $14(13-16)$ \\
\hline Adolescent idiopathic & $328(22)$ & $16(15-18)$ & $1188(78)$ & $15(13-16)$ & $1516(49)$ & $15(14-17)$ \\
\hline Neuromuscular & $465(51)$ & $15(13-17)$ & $448(49)$ & $13(12-16)$ & $913(30)$ & $14(12-16)$ \\
\hline MMC & $33(40)$ & $13(10-15)$ & $49(60)$ & $12(10-14)$ & $82(3)$ & $12(10-15)$ \\
\hline Congenital & $97(41)$ & $13(6-15)$ & $139(59)$ & $12(7-13)$ & $236(8)$ & $12(6-14)$ \\
\hline Unspecified $^{\mathrm{a}}$ & $36(49)$ & $15(13-18)$ & $38(51)$ & $14(12-17)$ & $74(2)$ & $15(12-17)$ \\
\hline
\end{tabular}

ancluding other secondary scoliosis and other forms of scoliosis

$I Q R$ inter quartile range, $M M C$ myelomeningocele

\section{Results}

The annual incidence of surgery regardless of type of scoliosis per 100,000 individuals was 9.1 [95\% confidence interval (CI), 8.8-9.4], 5.9 (95\% CI 5.5-6.2) in males and 12.5 (95\% CI 12.0-13.1) in females. The highest annual incidence was seen in females with adolescent idiopathic scoliosis 7.2 (95\% CI 6.8-7.7). The same type of scoliosis had the lowest incidence ratio between males and females, 0.3:1 (95\% CI 0.2-0.3). The male/female incidence ratio regardless of type of scoliosis was 0.5:1 (0.4-0.5) (95\% CI 0.4-0.5) (Table 2). The overall median age at admission was 15 years (IQR 13-17) for males and 14 years (IQR 12-16) for females. In total, incidence increased by 92\% (110\% in males and $90 \%$ in females), between 2000 and 2013, from 5.1 (95\% CI 4.2-6.0) to 10.7 (95\% CI 9.4-12.0) per 100,000 with an average of $4.6 \%$ per year $(p<0.001)$. The annual incidence of primary scoliosis surgery increased during the study period for both males $(r=0.88 ; p<0.001)$ and females $(r=0.77 ; p<0.001)$ (Fig. 1).

The trend in incidence rate was significant in adolescent idiopathic scoliosis $(r=0.85 ; p<0.001)$. The median length of hospital stay decreased continuously, in total by $27 \%$, from 11 days (IQR 9-14) in 2000-8 days (IQR 7-10) in 2013. Individuals with neuromuscular scoliosis had the longest LOS [median $=10($ IQR 9 to 14)] and individuals with adolescent idiopathic scoliosis had the shortest LOS [median $=9$ (IQR 8 to 10)], see Table 3 and Fig. 2.

Posterior fusion was the most common surgical approach $(n=2286 ; 75 \%)$ followed by anterior fusion $(n=546 ; 18 \%)$ and anteroposterior fusion $(n=230 ; 7 \%)$. There was a statistically significant shift towards the proportion of posterior fusion $(r=0.83 ; p<0.001)$ with a resultant decrease in anterior and anteroposterior fusion over the 14 years of surveillance (Table 3).

Of the 3062 individuals treated surgically for scoliosis, $3 \%(n=103)$ had revision surgery due to post-op infection
Table 2 Crude incidence rates for primary scoliosis surgery and incidence rate ratio for primary scoliosis surgery between males and females ages 0-21 years, in Sweden (2010-2013)

\begin{tabular}{|c|c|c|c|c|}
\hline \multirow[t]{2}{*}{ Type of Scoliosis } & \multicolumn{3}{|c|}{$\begin{array}{l}\text { Annual incidence rate per } 100,000 \text { ( } 95 \% \text { confidence } \\
\text { intervals) }\end{array}$} & \multirow{2}{*}{$\begin{array}{l}\text { Incidence rate ratio } \\
\text { (95\% Confidence } \\
\text { Intervals) } \\
\text { Male/female ratio }\end{array}$} \\
\hline & Males & Females & Total & \\
\hline Regardless of type & $5.9(5.5-6.2)$ & $12.5(12.0-13.1)$ & $9.1(8.8-9.4)$ & $0.5(0.4-0.5)$ \\
\hline Infantile idiopathic ${ }^{a}$ & $0.02(0-0.05)$ & $0.04(0.01-0.07)$ & $0.03(0.02-0.06)$ & $0.5(0.1-1.7)$ \\
\hline Juvenile idiopathic & $0.3(0.3-0.4)$ & $1.1(0.9-1.3)$ & $0.7(0.6-0.8)$ & $0.4(0.3-0.6)$ \\
\hline Adolescent idiopathic & $1.9(1.7-2.1)$ & $7.2(6.8-7.7)$ & $4.5(4.3-4.7)$ & $0.3(0.2-0.3)$ \\
\hline Neuromuscular & $2.7(2.5-2.9)$ & $2.7(2.5-3.0)$ & $2.7(2.5-2.9)$ & $1.0(0.9-1.1)$ \\
\hline MMC & $0.2(0.1-0.3)$ & $0.3(0.2-0.4)$ & $0.2(0.2-0.3)$ & $0.7(0.4-1.0)$ \\
\hline Congenital & $0.6(0.5-0.7)$ & $0.9(0.7-1.0)$ & $0.7(0.6-0.8)$ & $0.7(0.5-0.9)$ \\
\hline Unspecified $^{\mathrm{a}}$ & $0.2(0.1-0.3)$ & $0.2(0.1-0.3)$ & $0.2(0.2-0.3)$ & $1.0(0.6-1.6)$ \\
\hline
\end{tabular}

${ }^{\mathrm{a}} 95 \% \mathrm{CI}$ Rates for Infantile idiopathic scoliosis is based on very small number (4 males and 7 females) and are not reliable

${ }^{\mathrm{b}}$ Including other secondary scoliosis and other forms of scoliosis

$M M C$ myelomeningocele 


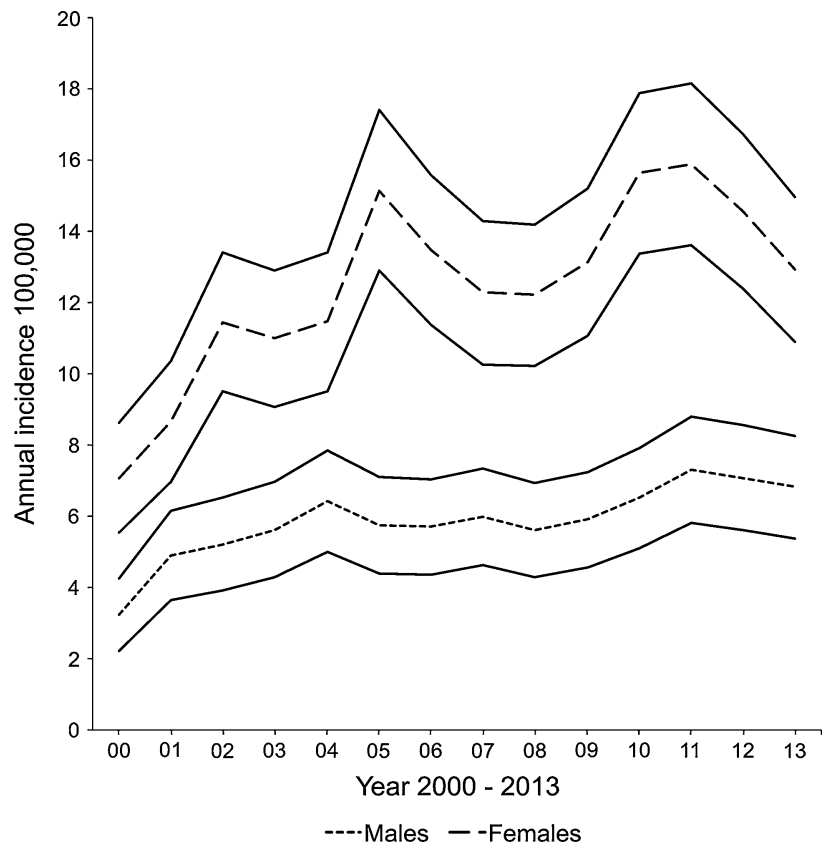

Fig. 1 Incidence of primary scoliosis surgery per 100,000 individuals, stratified by sex, as a function of calendar year of occurrence. The solid lines indicate $95 \%$ confidence intervals

(Table 3). Comparing revision surgery based on the time period prior to and including 2006 (56/1349) versus after $2006(47 / 1713)$, there was a 54\% increase in revision surgery $(\mathrm{RR}=1.54 ;$ 95\% CI 1.04-2.28) during 2000-2006. Sixteen $(0.5 \%)$ individuals died within 90 days after the primary scoliosis surgery. Individuals with neuromuscular scoliosis exhibited the highest mortality $(n=12 ; 1.3 \%)$.

\section{Discussion}

This nationwide study aimed to describe the annual incidence of surgical treatment of scoliosis in Swedish individuals and young adults. Specifically, we aimed to identify changes in incidence rate and hospital length of stay (LOS) over this 14 years period and to describe total infections and mortality within 90 days of surgery. There was a significant increase in annual surgeries for scoliosis between 2000 and 2013. The most common indication for surgery was adolescent idiopathic scoliosis. The increase in surgery among this subgroup may be due to the movement away from bracing as a result of difficulties with patient adherence, as well as the long-term psychological impact [14], and leading surgeons to recommend surgery more often [15]. At end of the time period examined in this study, Weinstein et al. demonstrated significant benefits of bracing for managing scoliosis using a randomized controlled trial [16]. Whether this study will have an impact on surgical rates remains to be seen. While

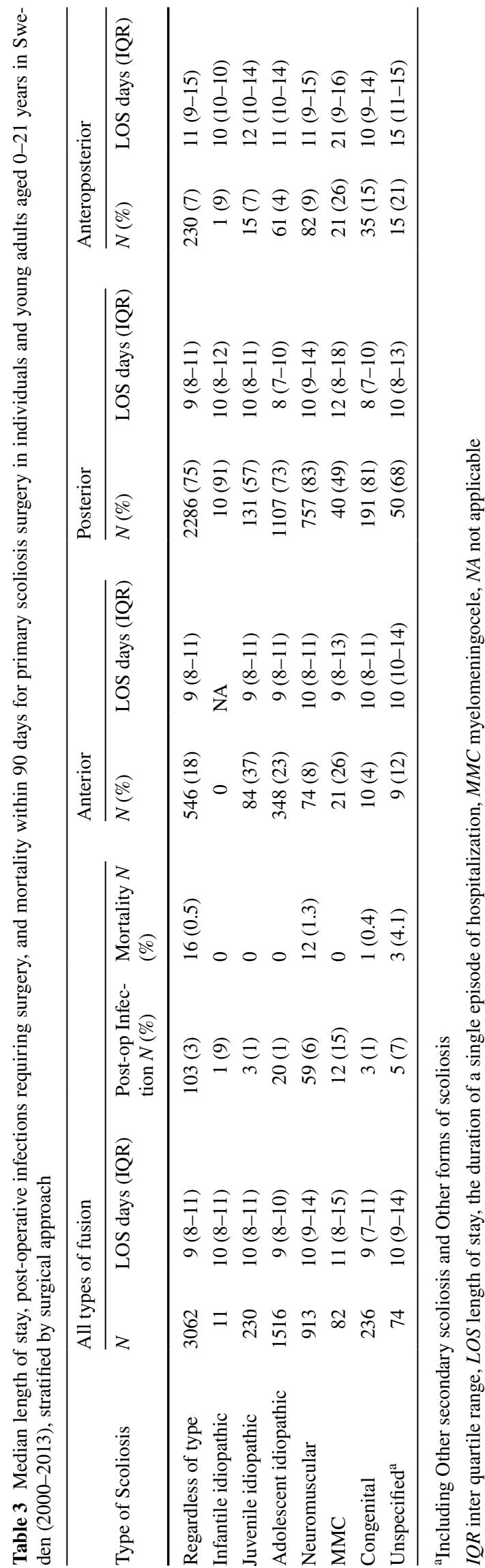




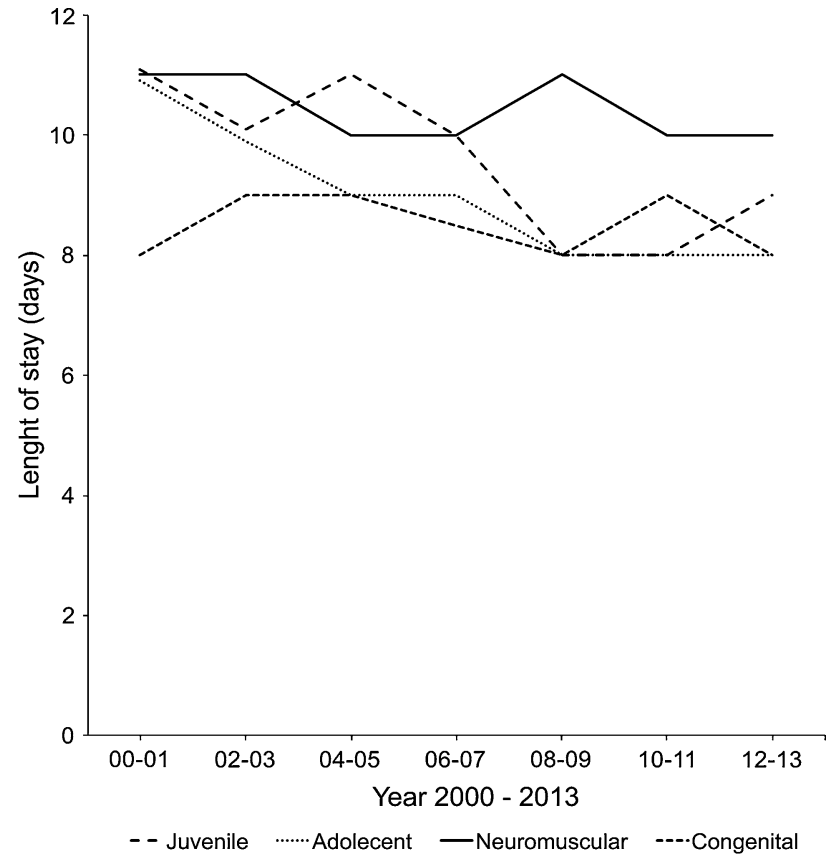

Fig. 2 Length of stay (days) stratified by type of scoliosis, as a function of calendar year of occurrence by periods of 2 years

we did not see a significant increase in surgery for persons with neuromuscular scoliosis, the shift from conservative treatment to surgical management of scoliosis in this group of Swedish children likely happened prior to 2000 [17]. There was no significant change in incidence of scoliosis surgery for children with MMC. One possible explanation for this lack of change is the fact that there are few cases of children with this condition. Despite the reduction in the number of Swedish children born with MMC due preventive measures (e.g., folic acid use during pregnancy and early detection of the condition in utero which has led to terminations of pregnancies) [18] there has been an influx of children with MMC into Sweden. We do not know if the prevalence of MMC changed during the study period. Additionally, surgical techniques may have advanced enough for the surgery to be considered safe among this group of children.

The surgical incidence for scoliosis was higher in females for all types of scoliosis, except for neuromuscular scoliosis and congenital scoliosis and these data confirm results from earlier studies $[7,17,19]$. When comparing our data to data from the 1970s, it seems that the sex differences for surgically treated adolescent idiopathic scoliosis are changing towards a higher proportion of males being treated than females [1]. With the information we have available in this study, we cannot provide insights into the shift towards proportionally more males receiving surgery.

While surgery increased during the study period, there was a concurrent decrease in hospital LOS over the 14-year period. These results mirror findings from other large population-based research demonstrating significant reductions in hospital LOS $[7,8,17,20]$. Vigneswaran et al. in a study of surgical outcomes in individuals with idiopathic scoliosis, reported a decrease in average hospital LOS from 6.5 days in 1997 to 5.6 days in 2012 [8], which does not seem to be associated with higher complication rates [21]. In a more recent study (2002-2011) of surgery in 2154 individuals with neuromuscular scoliosis, researchers reported a mean hospital LOS of 9.0 days [17].

With respect to surgical approach, posterior fusions significantly increased with a concurrent decrease in anteroposterior fusion over time. This trend was also found in the US among adolescents undergoing surgery for scoliosis $[7,8]$. Vigneswaran et al. in a study of 20,346 adolescents with scoliosis aged $0-12$ years, found posterior fusions increased from $63.4 \%$ in 1997 to $94.1 \%$ in 2012 [8]. The shift in surgical approach away from anterior fusions may be attributed to the re-popularization of the posterior approach and the increased pulmonary function described after posterior surgery compared to anterior surgery [22].

The number of individuals in our study with various types of scoliosis requiring revision surgery due to infection was similar to rates reported in earlier studies [17, 23-26]. However, infection rates reported in prior studies and our study cannot be directly compared with each other. Data on infection rates differ according to the definition of infections included (e.g., superficial infections requiring antibiotics, other studies only include infections which require revision surgery), and studies have analyzed infections rates within different time spans. A retrospective review of patients who were revised more than one year after the index procedure found that Titanium alloy instrumentations resulted in less late post-operative infections, when compared to stainlesssteel ones [27]. In Sweden there was a transition in the type of instrumentation used (stainless steel was the most common alloy in implants) prior to and including 2006 and after 2006 (titanium alloys were more common), we examined revision surgery rates and found a 54\% increase in revision surgery due to infections during the earlier time period when stainless steel was likely used. In our study, children with MMC experienced the highest post-operative infection rate. There are few studies that have evaluated surgical outcome, including infections for children with this type of scoliosis [28].

In this study, individuals with neuromuscular scoliosis exhibited the highest mortality (1.4\%). Mortality related to scoliosis surgery has been examined in a variety of ways. In a study of 8975 pediatric patients undergoing 29 different orthopedic procedures, four deaths were noted $(0.04 \%$ of all patients) within 30 days. These deaths occurred only in patients with non-idiopathic scoliosis $(n=655)$ [29]. De la Garza Ramos (2016) found a seemingly high mortality 
rate of $0.12 \%$ among 36,335 individuals with adolescent idiopathic scoliosis during the inpatient stay [20]. In our study there were no deaths among individuals with adolescent idiopathic scoliosis.

\section{Limitations and strengths}

Due to the retrospective nature of the study and the use of a national registry there is the potential for misclassification bias or missing data, as we did not confirm surgeries in the medical records of patients. However, the quality of the registry data has been systematically reviewed and the accuracy of the coding is reported to be high [30]. However, we cannot exclude that the increase of incidence of scoliosis surgeries might be affected by an increased awareness of the medical system towards this diagnosis. As it is impossible to ascertain the number of individuals diagnosed with scoliosis from the registry data, our focus is on the changes in surgical management within the total population at risk. It is likely that surgical technique has changed over time, but we find it unlikely that this have resulted in different reporting patterns to the National Patient Register. A strength of the study includes examination surgical management of scoliosis within a national registry embedded in a public health system. Thus, we have complete ascertainment of the population of risk for surgeries every year. To our knowledge, this is the largest epidemiology study of surgical management for individuals and adolescents with scoliosis to examine various types of scoliosis and trends of this management in a national population. There were no changes in diagnostic codes during the time period of the study (all have ICD10 codes). To minimize the possibility of including children who had scoliosis surgery prior to the study period, we examined the database and excluded children who had surgery between 1997 and 2000.

\section{Conclusions}

During the study period 2000-2013, the incidence of surgical treatment of scoliosis in individuals and adolescents increased, driven primarily by increases in surgical management for adolescent scoliosis. We do not expect this increase to reflect a change in surveillance techniques. Rather, we postulate that surgeons were more likely to recommend surgery for scoliosis due to improvements in surgical techniques, better peri- and post-operative care, and reduced bleeding time and complications, even though we lack data to support this assumption.

The decrease in LOS may reflect improvements in surgical techniques and post-operative care. This present nationwide study is the largest report focused on trends in the surgical management of individuals and adolescents with scoliosis diagnosed with various types of scoliosis and may serve as a basis for further studies.

\section{Compliance with ethical standards}

Funding This study was financially supported by funds from the Swedish Research Council (number K-2013-52X-22,198-01-3), the regional agreement on medical training and clinical research (ALF) between Stockholm County Council and Karolinska Institutet. Dr. Iversen is supported by NIAMS-MCRC: \#P60 AR047782 and R01 AR059086 and by a US Fulbright Award. The contents of this paper are solely the responsibility of the authors and do not represent official views of the US government or its agencies.

Conflict of Interest Conflict of Interest: Johan von Heideken, Maura D Iversen, and Paul Gerdhem declare that they have no conflict of interest.

Open Access This article is distributed under the terms of the Creative Commons Attribution 4.0 International License (http://creativecommons.org/licenses/by/4.0/), which permits unrestricted use, distribution, and reproduction in any medium, provided you give appropriate credit to the original author(s) and the source, provide a link to the Creative Commons license, and indicate if changes were made.

\section{References}

1. Willner S, Uden A (1982) A prospective prevalence study of scoliosis in Southern Sweden. Acta Orthop Scand 53:233-237

2. Shands AR Jr, Eisberg HB (1955) The incidence of scoliosis in the state of Delaware; a study of 50,000 minifilms of the chest made during a survey for tuberculosis. J Bone Joint Surg Am 37:1243-1249

3. Hedequist D, Emans J (2007) Congenital scoliosis: a review and update. J Pediatr Orthop 27:106-116. doi:10.1097/ BPO.0b013e31802b4993

4. Madigan RR, Wallace SL (1981) Scoliosis in the institutionalized cerebral palsy population. Spine 6:583-590

5. Sarwark J, Sarwahi V (2007) New strategies and decision making in the management of neuromuscular scoliosis. Orthop Clin North Am 38:485-496. doi:10.1016/j.ocl.2007.07.001

6. Kim HJ, Blanco JS, Widmann RF (2009) Update on the management of idiopathic scoliosis. Curr Opin Pediatr 21:55-64. doi:10.1097/MOP.0b013e328320a929

7. Theologis AA, Sing DC, Chekeni F, Diab M (2017) National trends in the surgical management of adolescent idiopathic scoliosis: analysis of a National estimate of 60,108 children from the National inpatient sample over a 13-year time period in the United States. Spine Deform 5:56-65. doi:10.1016/j.jspd.2016.09.001

8. Vigneswaran HT, Grabel ZJ, Eberson CP, Palumbo MA, Daniels AH (2015) Surgical treatment of adolescent idiopathic scoliosis in the United States from 1997 to 2012: an analysis of 20,346 patients. J Neurosurg Pediatr 16:322-328. doi:10.3171/2015.3.P EDS14649

9. The Swedish National Board of Health and Welfare, The Swedish Inpatient Register. http://www.socialstyrelsen.se/register/halsodataregister/patientregistret/inenglish. Accessed 15 Jan 2017

10. The Swedish National Board of Health and Welfare, ICD-10 SE. http://www.socialstyrelsen.se/klassificeringochkoder/diagnoskodericd-10. Accessed 15 Jan 2017 
11. The Swedish National Board of Health and Welfare, Swedish Classification of Surgical Procedures 1997; 2004. http://www. socialstyrelsen.se/publikationer2004/2004-4-1. Accessed 15 Jan 2017

12. The Swedish National Board of Health and Welfare, Cause of Death Register. http://www.socialstyrelsen.se/statistics/statisticaldatabase/help/causeofdeath. Accessed 15 Jan 2017

13. Statistics Sweden Population statistics. http://www.scb.se/en/ finding-statistics/statistics-by-subject-area/population/populationcomposition/population-statistics/. Accessed 15 Jan 2017

14. Danielsson AJ, Wiklund I, Pehrsson K, Nachemson AL (2001) Health-related quality of life in patients with adolescent idiopathic scoliosis: a matched follow-up at least 20 years after treatment with brace or surgery. Eur Spine J 10:278-288

15. Negrini S (2008) Approach to scoliosis changed due to causes other than evidence: patients call for conservative (rehabilitation) experts to join in team orthopedic surgeons. Disabil Rehabil 30:731-741. doi:10.1080/09638280801889485

16. Weinstein SL, Dolan LA, Wright JG, Dobbs MB (2013) Effects of bracing in adolescents with idiopathic scoliosis. N Engl J Med 369:1512-1521. doi:10.1056/NEJMoa1307337

17. Rumalla K, Yarbrough CK, Pugely AJ, Koester L, Dorward IG (2016) Spinal fusion for pediatric neuromuscular scoliosis: national trends, complications, and in-hospital outcomes. J Neurosurg Spine 25:500-508. doi:10.3171/2016.2.SPINE151377

18. Clemmensen D, Thygesen M, Rasmussen MM, Fenger-Gron M, Petersen OB, Mosdal C (2011) Decreased incidence of myelomeningocele at birth: effect of folic acid recommendations or prenatal diagnostics? Childs Nerv Syst 27:1951-1955. doi:10.1007/s00381-011-1473-2

19. Ersberg A, Gerdhem P (2013) Pre- and postoperative quality of life in patients treated for scoliosis. Acta Orthop 84:537-543. doi $: 10.3109 / 17453674.2013 .854667$

20. De la Garza Ramos R, Goodwin CR, Abu-Bonsrah N, Jain A, Miller EK, Huang N, Kebaish KM, Sponseller PD, Sciubba DM (2016) Patient and operative factors associated with complications following adolescent idiopathic scoliosis surgery: an analysis of 36,335 patients from the Nationwide Inpatient Sample. J Neurosurg Pediatr 25:730-736. doi:10.3171/2016.6.PEDS16200

21. Fletcher ND, Shourbaji N, Mitchell PM, Oswald TS, Devito DP, Bruce RW (2014) Clinical and economic implications of early discharge following posterior spinal fusion for adolescent idiopathic scoliosis. J Child Orthop 8:257-263. doi:10.1007/ s11832-014-0587-y
22. Lee AC, Feger MA, Singla A, Abel MF (2016) Effect of surgical approach on pulmonary function in adolescent idiopathic scoliosis patients: a systemic review and meta-analysis. Spine (Phila Pa 1976) 41:E1343-E1355. doi:10.1097/brs.0000000000001619

23. Geiger F, Parsch D, Carstens C (1999) Complications of scoliosis surgery in children with myelomeningocele. Eur Spine J 8:22-26

24. Divecha HM, Siddique I, Breakwell LM, Millner PA, British Scoliosis Society M (2014) Complications in spinal deformity surgery in the United Kingdom: 5-year results of the annual British Scoliosis Society National Audit of Morbidity and Mortality. Eur Spine J 23(Suppl 1):S55-60. doi:10.1007/s00586-014-3197-y

25. Smith JS, Shaffrey CI, Sansur CA, Berven SH, Fu KM, Broadstone PA, Choma TJ, Goytan MJ, Noordeen HH, Knapp DR Jr, Hart RA, Donaldson WF 3rd, Polly DW Jr, Perra JH, BoachieAdjei O, Scoliosis Research Society Morbidity and Mortality Comittiee (2011) Rates of infection after spine surgery based on 108,419 procedures: a report from the Scoliosis Research Society Morbidity and Mortality Committee. Spine (Phila Pa 1976) 36:556-563. doi:10.1097/BRS.0b013e3181eadd41

26. Reames DL, Smith JS, Fu KM, Polly DW Jr, Ames CP, Berven SH, Perra JH, Glassman SD, McCarthy RE, Knapp RD Jr, Heary R, Shaffrey CI, Scoliosis Research Society Morbidity and Mortality Committee (2011) Complications in the surgical treatment of 19,360 cases of pediatric scoliosis: a review of the Scoliosis Research Society Morbidity and Mortality database. Spine (Phila Pa 1976) 36:1484-1491. doi:10.1097/BRS.0b013e3181f3a326

27. Di Silvestre M, Bakaloudis G, Lolli F, Giacomini S (2011) Late-developing infection following posterior fusion for adolescent idiopathic scoliosis. Eur Spine J 20(Suppl 1):S121-S127. doi:10.1007/s00586-011-1754-1

28. Mummareddy N, Dewan MC, Mercier MR, Naftel RP, Wellons JC 3rd, Bonfield CM (2017) Scoliosis in myelomeningocele: epidemiology, management, and functional outcome. J Neurosurg Pediatr. doi: 10.3171/2017.2.PEDS16641

29. Basques BA, Lukasiewicz AM, Samuel AM, Webb ML, Bohl DD, Smith BG, Grauer JN (2015) Which pediatric orthopaedic procedures have the greatest risk of adverse outcomes? J Pediatr Orthop. doi:10.1097/BPO.0000000000000683

30. Ludvigsson JF, Andersson E, Ekbom A, Feychting M, Kim JL, Reuterwall C, Heurgren M, Olausson PO (2011) External review and validation of the Swedish national inpatient register. BMC Public Health 11:450. doi:10.1186/1471-2458-11-450 